\title{
The Crisis of Opiates in Appalachia
}

The opiate abuse crisis gripping the Appalachian region is one of the most challenging health issues of our time. Serious consequences resulting from this problem are of staggering proportions. While overdose deaths and crime lead the headlines, other consequences are equally concerning. The number of children born exposed to opiates has skyrocketed, and we have yet to understand the long-term consequences. Hepatitis rates have exploded due to intravenous drug use. Young adults with felony drug convictions can't find employment. This problem has shredded the family structure in our communities. This is a frightening reality.

Although the opiate crisis is not new to Appalachia, it is evolving in a dangerous ways such as the introduction of black tar heroin in our region. An excellent account of the socioeconomic factors driving this devastating social phenomenon is well described in "Dreamland, the True Tale of America's Opiate Epidemic” by Sam Quinones: a book I strongly recommend. Quinones traces the introduction of heroin by Mexican growers to the Appalachian region and explores the complex social and economic factors that account for the problems explosive expansion.

Addiction is one of the most challenging health conditions to manage. The problem's complications include the combination of physical and mental health diagnoses that interact with social factors, leading vulnerable individuals to self-medicate with narcotics. It is common for addicts to have experienced traumatic life events. Many have histories of criminal activity. Even if treatment addresses all of these factors driving the addiction, a patient may move toward recovery, fail, and relapse into drug use again, and then recycle through the treatment programs. The deadly cycle often repeats time and again. Unlike most medical conditions that can be cured or, at least stabilized, addiction frequently runs out of control with devastating impact on addicts, families and communities. Addiction medicine treatment specialists recognize the instability of the condition and learn to accept small successes. For the addicted patient, success may be as simple as 3 months being drug free, reconnecting with family, or having stable housing. It is a difficult and tragic life.

In the 1960s and 70s, America confronted heroin addiction in soldiers returning from the Vietnam War. One of the responses to this address this problem was the War on Drugs and the adoption of medication-assisted treatment (MAT) programs. These were primarily based on using methadone as a maintenance medication. Studies performed in those years demonstrated that, for individuals with refractory opiate dependence, maintenance with daily methadone could enable the patient to maintain employment and fully engage in society. Today, MAT continues to be a mainstay of treatment programs aimed at managing addiction. Contemporary treatment programs rely on a variety of medications including methadone, buprenorphine/naloxone combinations, or naltrexone. Many questions concerning this treatment approach remain unasked and unanswered. Which patients are most appropriate for MAT with which medication? What criteria should guide the choice of this therapy? Is MAT a life-long treatment approach? Would MAT treatment be effective in rural settings where limited employment options are an additional complication? 
Decades ago "abstinence programs" emerged as a treatment for alcohol abuse. These became known as "12-step" or Alcoholics Anonymous (AA) programs. Today, excellent abstinence programs for narcotics represent another important option for the treatment of opiate addiction. These programs frequently offer peer-support and assistance in dealing with the myriad social consequences of addiction. These programs are available to addicts without cost and they are voluntary and anonymous, a fact that has limited the opportunity to collect data on effectiveness in reducing addictive behavior.

In addition to treatment programs, other approaches have been implemented to reduce the impact of adverse health effects resulting from opiate abuse. "Harm reduction" efforts in the form of naloxone programs and needle exchanges are being created in many county Health Departments. These programs can help to reduce overdose deaths and the spread of viral hepatitis and HIV. Health Departments also offer a hepatitis B vaccine for people at high risk due to IV drug use.

When providers work with drug-addicted patients they often discover gaps in the evidence-based findings that should guide the management of opiate addiction. There are clinical guidelines from the Substance Abuse and Mental Health Services Administration and the National Institute on Drug Abuse. A comprehensive look at substance use disorder and its treatment was published in 2014 by The Institute for Clinical and Economic Review. This comparative effectiveness review outlines the evidence for treatment approaches in substance use disorder. It is an important read for providers and policymakers as they tackle this crisis. Despite the availability of these excellent resources many important questions remain to be explored, especially in select population and situations. In my opinion the significance of the opiate crisis in Appalachia establishes an important opportunity, perhaps an obligation, to engage in further systematic studies of the phenomenon of addiction in rural populations.

James Becker, MD

\section{References:}

1. Quinones, S. Dreamland, the True Tale of America's Opiate Epidemic. Bloomsbury Press, April 2015.

2. ICER, Management of Patients with Opioid Dependence: A Review of Clinical, Delivery System and Policy Options. The New England Comparative Effectiveness Public Advisory Council Public Meeting June, 2014. 